EPJ Web of Conferences 59, 18004 (2013)

DOI: $10.1051 /$ epjconf/20135918004

(C) Owned by the authors, published by EDP Sciences, 2013

\title{
Scattering of relativistic electron beam by two counter-propagating laser pulses: A new approach to Raman X-ray amplification
}

\author{
I.A. Andriyasha , Ph. Balcou, E. d'Humières and V.T. Tikhonchuk \\ Centre Lasers Intenses et Applications, Université Bordeaux 1-CNRS-CEA, 33405 Talence, \\ France
}

\begin{abstract}
We present a detailed study of the properties of electron beam injected and trapped in an high intensity optical lattice. By using the hydrodynamic and kinetic approaches, we identified the beam trapping conditions, the high-frequency longitudinal beam eigenmodes and their dependence on the electron angular and energy spread. The coupling of these beam eigenmodes to the laser waves is also considered. This corresponds to the convective parametric instability: a stimulated scattering of two laser beams creating the optical lattice on the trapped electron beam mode. The amplification coefficients for the up-scattered Raman modes propagating parallel to the electron beam are calculated and their dependence on the beam characteristics is analyzed.
\end{abstract}

\section{INTRODUCTION}

The study of relativistic electron beams collective behaviour in a guiding and wiggling field has become a common interest for accelerator physics, plasma physics, nonlinear optics and laser science. By wiggling relativistic charged particles one may produce a scattered electromagnetic wave with a wavelength much shorter than the spatial period of the wigglers. This process lays at the basis of a number of techniques of XUV, X-ray and $\gamma$-ray generation, which find numerous applications in the diagnostics from medical physics to fusion science.

Here we present a new approach to XUV/X-ray generation, based on the scattering of the bunch of relativistic electrons by a specific electromagnetic structure, called high intensity optical lattice. Such a field is created by the interference of two coherent laser pulses of equal frequencies and intensities, and it represents a standing wave pattern, where charged particles can be trapped. The electron oscillations in such a ponderomotive potential can be coupled to the laser wave field and emitted as high- and lowfrequency electromagnetic waves. This process can be employed for generation of coherent radiation in a broad wavelength range extending from the infrared to X-ray domain [1].

One of the promising applications of this technique is Raman XUV/X-ray amplification using a relativistic electron beam, propagating through an optical lattice [2, 3]. The conventional schemes of backscattering amplification in stationary wigglers impose unrealistically severe limitations on the quality (angular and velocity spread) of the electron beam [4, 7]. Raman amplifiers with optical lattices even for moderate laser intensities open a possibility to reduce strongly these limitations and to use laser accelerated electron bunches thus paving a way for compact all-optical systems.

\footnotetext{
ae-mail: andriyash@celia.u-bordeaux1.fr
}

This is an Open Access article distributed under the terms of the Creative Commons Attribution License 2.0, which permits unrestricted use, distribution, and reproduction in any medium, provided the original work is properly cited. 
Table 1. Conversion of the parameters between the laboratory and electron beam reference systems. The current $J$ is expressed in amperes [A]; the normalized emittance and transverse size of the beam $\epsilon_{N}^{l}[\mathrm{~mm} \cdot \mathrm{mrad}]$ and $\sigma_{\perp}^{l}[\mu \mathrm{m}]$; $a=e A / m c^{2}$ stands for the dimensionless vector potential and $I^{l}\left[\mathrm{~W} / \mathrm{cm}^{2}\right]$ and $\lambda_{0}^{l}[\mu \mathrm{m}]$ are laser the intensity and wavelength.

\begin{tabular}{ll}
\hline beam & $n_{b}=4 \cdot 10^{16} \cdot J /\left(\gamma_{0} \sigma_{\perp}^{l 2}\right), \mathrm{cm}^{-3} ; \delta \beta_{\perp 0}=\gamma_{0} \delta \beta_{\perp 0}^{l}=\epsilon_{N}^{l} /\left(\beta_{0} \sigma_{\perp}^{l}\right) ; \delta \beta_{\| 0}=\delta \gamma_{\|}^{l} /\left(\gamma_{0} \beta_{0}\right)$ \\
laser light & $\omega_{0}=\gamma_{0} \omega_{0}^{l} ; k_{0 \perp}=k_{0}^{l} ; k_{0 \|}=\gamma_{0} \beta_{0} k_{0}^{l} ; a_{0}=0.85 \cdot 10^{-9} \lambda_{0}^{l} \sqrt{I^{l}} ; a_{x}=-\beta_{0} a_{z}^{l} ; a_{y}=a_{y}^{l} ; a_{z}=a_{z}^{l} / \gamma_{0}$ \\
\hline
\end{tabular}

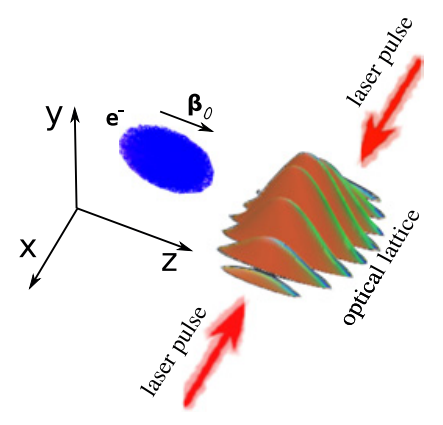

Figure 1. Laser-beam interaction geometry in laboratory reference system.

\section{INJECTION AND TRAPPING OF ELECTRON BEAM IN THE OPTICAL LATTICE}

The electron beam is injected into the interference pattern created by two counter-propagating laser waves. The phase-space distribution of the trapped electrons is shown in Fig. 1. The optical lattice consists of a first ramp of intensity over one to few millimeters $l_{\text {ramp }}$, followed by a stage of a constant intensity.

The problems of interaction of relativistic electrons with electromagnetic waves can be considered in the reference system, where the electron beam is at rest. In such a frame, the structure of electromagnetic field is modified due to the Lorentz transformation and represents the actual field, that the electrons "see". Injection of electrons into the lattice corresponds to a growth of the external field from $a(0)=0$ to $a(\tau)=a_{0}$ during the time $\tau=l_{\text {ramp }} / \gamma_{0} \beta_{0} c$. The total field created by interference of two laser beams reads: $a_{L}=2 a(t) \sin \left(k_{0 \perp} x\right) \cos \left(\omega_{0} t+k_{0 \|} z\right)$, where the origin of $x$-axis is chosen in the node of the field. For the laser intensities much less than $10^{18} \mathrm{~W} / \mathrm{cm}^{2}$, the electron motion in the beam reference frame may be described by a non-relativistic model and studied within a classic collisionless kinetic approach. Considering a long electron beam, homogeneous along its axis, we describe injection with help of the Vlasov kinetic equation keeping only the terms associated with a motion along the $x$-axis (see Fig. 1):

$$
\partial_{t} f+v_{x} \partial_{x} f-\partial_{x} U(x, t) \partial_{v_{x}} f=0,
$$

where function $U(x, t)=U_{p}(x, t)-e \phi / m$ defines the effective potential, which includes the ponderomotive potential $U_{p}(x, t)=-\Omega^{2} \cos ^{2}\left(k_{0 \perp} x\right) / 2 k_{0 \perp}^{2}$, where $\Omega(t)=\sqrt{2} a(t) k_{0 \perp} c$ is a frequency of the small-amplitude electron oscillations in this potential. The ratio between the ponderomotive and the Coulomb potential $\phi$ scales as $\Omega_{0} / \omega_{p}$, where $\omega_{p}=\left(4 \pi e^{2} n_{b} / m\right)^{1 / 2}$ is a plasma frequency and $\Omega_{0}=\Omega(\tau)$. Considering parameters $\Omega_{0}^{-1}, \tau \ll \omega_{p}^{-1}$ one may neglect the Coulomb potential in first approximation.

If the injection time is shorter than the time of electron propagation to the edge of potential $\langle\Delta t\rangle$, we consider the fast injection process and otherwise we call it slow. Considering a linear ramp, $a=a_{0}(t / \tau)$ 


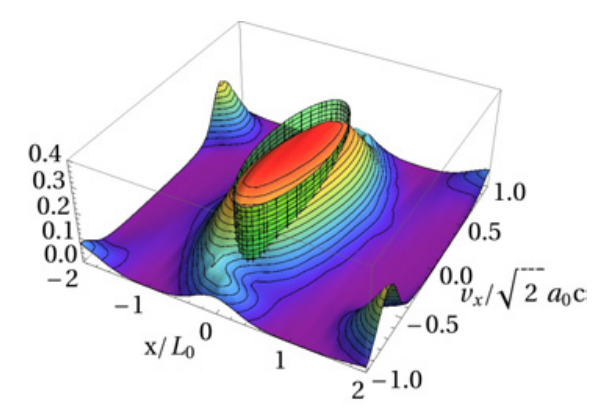

Figure 2. Normalized electron distribution function (EDF) of the trapped beam in the $\left(x, v_{x}\right)$ plane for initial velocity spread $\delta \beta_{\perp 0} / \sqrt{2} a_{0} c=1$ and injection time $\tau=5 \Omega_{0}^{-1}$. The green cylinder shows the localization region given by model EDF, Eq. (2).

for $t<\tau$, we find the maximum initial velocity of trapped electrons:

$$
v_{\perp m} / c=\left[\begin{array}{ll}
\operatorname{Min}\left(\delta \beta_{\perp 0}, \sqrt{2} a_{0}\right), & \langle\Delta t\rangle>\tau, \\
\operatorname{Min}\left(\delta \beta_{\perp 0}, \sqrt{2} a_{0}, \sqrt{2} a_{0}\left(\Omega_{0} \tau\right)^{-1 / 2}\right),\langle\Delta t\rangle<\tau,
\end{array}\right.
$$

which defines, which particles may be trapped. The trapping duration $\tau_{t r}$ is considered as a moment, when the growing potential amplitude reaches the maximum kinetic energy of the particle and is estimated as $\tau_{t r}=v_{\perp m} \tau /\left(\sqrt{2} a_{0} c\right)$.

Assuming initially homogeneous distribution of electrons in $\left(x, v_{x}\right)$ phase plane, the electron distribution function (EDF) of the trapped beam at $t=\tau_{t r}$ reads:

$$
f_{t r}\left(v_{x}, x, t=\tau_{t r}\right)=\left(n_{b} / 2 c \delta \beta_{\perp 0}\right) \eta\left(v_{\max }(x)-\left|v_{x}\right|\right),
$$

where $\eta$ is a unit-step Heaviside function, and the maximum electron velocity is $v_{\max }(x)=v_{\perp m} \cos k_{\perp 0} x$. Thus, the phase region, bounded by the trapped particle trajectory, reads $\left(v_{x} / v_{\perp m}\right)^{2}+\sin ^{2} k_{\perp 0} x \leq 1$ and has a good agreement with a kApchinsky-Vladimirsky model (K-V distribution) [6].

The area of localization phase region represents the adiabatic invariant $I=\oint_{\mathbb{G}} v_{x} \mathrm{~d} x$ and remains constant for a slowly changing finite trajectory $\mathbb{G}$. Considering its conservation, we may describe the compressed state of the trapped beam by finding modified beam width $\sin k_{0 \perp} L(t)=\sqrt{\tau_{t r} / \tau}$ and a velocity amplitude $v_{\perp m}=\sqrt{2} a_{0} c \sqrt{\tau_{t r} / \tau}$. The density distribution of the electrons in the potential reads:

$$
n_{t r}(x)=n_{0}\left(1-2\left(a_{0} / \delta \beta_{\perp}\right)^{2} \sin ^{2} k_{\perp 0} x\right)^{1 / 2},
$$

where $n_{0}=n_{b} \delta \beta_{\perp} / \delta \beta_{\perp 0}$ is a maximum density and $\delta \beta_{\perp}=v_{\perp m} / c$.

This simplified description can be compared with the results of numerical simulations as it is presented in Fig. 2. It demonstrates the agreement of the trapping description given by Eq. (2) and beam subsequent compression (green cylinder) with the result of one-dimensional simulation of the particles motion in the growing ponderomotive potential.

\section{EIGENMODES OF TRAPPED ELECTRON BEAM}

The dynamics of the trapped beam is studied in the fluid approach, which comprises the equations for two first moments of the EDF and the Poisson equation:

$$
\partial_{t} n+\nabla \cdot(n \mathbf{u})=0, \quad \partial_{t} u_{i}+\nabla_{j} P_{i j} / m n+\nabla_{i}\left(U_{p}-e \phi / m\right)=0, \quad \nabla^{2} \phi=4 \pi e n,
$$




\section{EPJ Web of Conferences}

Here the components of pressure tensor are related to the density, as $P_{x x}=m v_{\perp}^{2} n^{3} / 3 n_{0}^{2}, P_{z z}=m v_{\|}^{2} n / 3$, $P_{y y}=m v_{\perp 0}^{2} n / 3$, where $v_{\|}=c \delta \beta_{\| 0}$, thus, the electrons follow the adiabatic equation of state in the $x$-direction and isothermal for the $y$ - and $z$-directions.

Considering the equilibrium solution and moderate electron density one may study the effect of the Coulomb repulsion on the density and find a condition for the maximum electron density that could be confined by the optical lattice:

$$
n_{e}^{l} / n_{c}^{l}<2 \gamma_{0}\left(a_{0}^{2}-\left(2 / \pi^{2}\right) \delta \beta_{\perp}^{2}\right) .
$$

For a low density beam, distribution (3) may be simplified and used to describe the density perturbations. We present them here as the temporal and spatial variations of the beam width $\Delta_{L}(z, t)$ and its centroid position $\Delta_{x}(z, t)$ in a self-similar form, $n=n_{0} \sqrt{1-\xi^{2}}\left(1-\Delta_{L} / L\right)$, where the dimensionless coordinate $\xi=\left(x+\Delta_{x}\right) /\left(L+\Delta_{L}\right)$. The harmonic oscillations of the beam position $\Delta_{x}$ and width $\Delta_{L}$ can be studied independently and define symmetric and anti-symmetric modes:

$$
\omega_{1}^{2}=\Omega_{0}^{2}+k^{2}\left(v_{\|}^{2} / 3+\omega_{p}^{2} L_{0}^{2}\right), \quad \omega_{2}^{2}=4 \Omega_{0}^{2}-2 \omega_{p 0}^{2}+k^{2}\left(v_{\|}^{2} / 3+\omega_{p}^{2} L_{0}^{2}\right) .
$$

at the first $\omega_{1}$ and second $\omega_{2}$ harmonics of the principal bounce frequency $\Omega_{0}$. These longitudinal oscillations are governed mainly by the ponderomotive force, while the effects of the self-consistent field and thermal motion are weaker. The eigenfrequencies are shifted due to the electron motion along $z$-direction defined by the initial velocity spread and Coulomb expansion.

Adding Maxwell equation $\left(\left(\partial_{t}-i \omega_{0}\right)^{2}-c^{2} \nabla^{2}\right) \bar{a}=-\left(4 \pi e^{2} / m\right) n_{t r} \bar{a}$ to the set of fluid equations Eq. (4) and accounting for the scattered wave $\delta a$ in a ponderomotive potential $U_{p}=(c / 2)^{2}|\bar{a}|^{2}$ one may study the coupling of the electromagnetic and beam modes in a linear approximation. The dispersion equation of anti-symmetric mode, coupled to a pump and scattered radiation, reads:

$$
\left(\omega^{2}-\Omega^{2}-k_{z}^{2} v_{\|}^{2} / 3\right)\left(\left(\omega-\omega_{0}\right)^{2}-\left(k_{z}-k_{0}\right)^{2} c^{2}-\tilde{\omega}_{p}^{2}\right) \simeq \omega_{p}^{2} \Omega^{2}\left(k_{z} L\right)^{2}\left\langle\xi^{2} \sqrt{1-\xi^{2}}\right\rangle .
$$

The factor in the angle brackets in Eq. (7), denotes the averaged value of the function $\xi^{2} \sqrt{1-\xi^{2}}$, which is due to the fact that the localizations of the beam and electromagnetic modes do not exactly match, and can be estimated integrally as $\simeq \pi / 16$.

Defining the growth rate of SRS as $\Gamma=\operatorname{Im}[\omega]$ and considering the condition, $\Gamma>\omega_{0} / \gamma_{b}^{2}$, we may neglect the diffraction of scattered wave within the bunch. Considering the width of the beam to be same as the channel width and the resonance of beam mode with electromagnetic ones for $k_{z} \simeq 2 k_{0}$, one may estimate the growth rate:

$$
\Gamma_{1} \simeq(\pi \sqrt{\pi} / 8) \omega_{p} \gamma_{b} \sqrt{\Omega / \omega_{0}} .
$$

The growth rate of the symmetric mode has the same order of magnitude $\Gamma_{2}=(2 / \sqrt{3}) \Gamma_{1}$.

To show the interest of this approach, one may note, using the latter formula, that a bunch of $10 \mathrm{MeV}$ electrons, with a current of $3 \mathrm{kA}$, concentrated over a $10 \mu \mathrm{m}^{2}$, irradiated by a Ti:Sa laser at $10^{17} \mathrm{~W} / \mathrm{cm}^{2}$, would amplify a beam of X-ray photons $(\mathrm{h} v=1.2 \mathrm{keV})$ with a gain length of 400 micrometers.

The obtained values of linear growth rates are derived without account for damping of the beam modes associated with the electron thermal motion. In general, the contribution of the thermal motion is given by the expansion of electron bunch, which drives the spatial modulation of electron density out of the resonance with electromagnetic field, thus preventing the instability growth. On the other hand thermal motion is responsible for Landau damping of plasma waves, when the energy of electrostatic field is resonantly converted to kinetic energy of electrons, while they propagate with a wave. The latter process presents one of the main obstacles in achievement of Raman amplification of X-ray [5] for the realistic beam quality. Considering this, the advantage of the SRS on the bounce mode, discussed here, is that the mode frequency can be controlled by the pump intensity and electron energy, and it is typically much higher than the electron plasma frequency $\Omega_{0} / \omega_{p} \propto a_{0}^{-1}\left(n_{e}^{l} / n_{c}^{l} \gamma_{b}\right)^{1 / 2} \ll 1$. 


\section{IFSA 2011}

Consequently, the SRS on the trapped beam mode, has a higher growth rate than the standard one on the plasma mode. Moreover, the growth rate increases faster with the beam density. These facts open a possibility to use laser accelerated electron bunches in a compact system for more efficient and tunable generation of X-rays.

\section{References}

[1] M. V. Fedorov, K. B. Oganesyan, and A. M. Prokhorov, Appl. Phys. Let. 53, 353 (1988)

[2] Ph. Balcou, Europ. Phys. Journ. D 59, 525 (2010)

[3] I.A. Andriyash, Ph. Balcou and V.T. Tikhonchuk, Europ. Phys. Journ. D 65, 533 (2011)

[4] P. Sprangle, B. Hafizi, J. R. Peñano, Phys. Rev. ST AB 12, 050702 (2009)

[5] P. Sprangle and A. T. Drobot, J. Appl. Phys. 50, 2652 (1979)

[6] M. Reiser, Theory and Design of Charged Particle Beams (Wiley-VCH, Weinheim, Germany, 2008)

[7] V. Petrillo, L. Serafini, P. Tomassini. Phys. Rev. ST AB 11, 070703 (2008) 